\title{
Small-Cell Lung Cancer Treatment of Newly Diagnosed Patients with Poor Performance Status
}

\author{
Yuka Aida ${ }^{a, b}$ Kensuke Nakazawa ${ }^{a}$ Toshihiro Shiozawa ${ }^{a}$ Ryoko Ogawa $^{a}$ \\ Takumi Kiwamoto $^{a}$ Yuko Morishima ${ }^{a}$ Toru Sakamoto ${ }^{a} \quad$ Ikuo Sekine ${ }^{b}$ \\ Nobuyuki Hizawa ${ }^{a}$ \\ aDepartment of Respiratory Medicine, Faculty of Medicine, University of Tsukuba, \\ Tsukuba, Japan; bepartment of Medical Oncology, Faculty of Medicine, University of \\ Tsukuba, Tsukuba, Japan
}

\section{Keywords}

Small-cell lung cancer · Poor performance status · Platinum · Chemotherapy · Supportive care

\begin{abstract}
Small-cell lung cancer (SCLC) is highly sensitive to platinum-based chemotherapy. However, its indication in patients with a poor performance status (PS) at initial diagnosis is controversial. We retrospectively reviewed all clinical courses of pathologically diagnosed SCLC patients with poor PS, Eastern Cooperative Oncology Group PS 3 and 4. Among 18 patients, 12 were treated with chemotherapy and 6 with supportive care alone. During the chemotherapy courses, PS improved in 7 (58.3\%, including the PS 4 cases), remained stable in $2(16.7 \%)$, and deteriorated in $3(25 \%)$ patients. Moreover, 5 patients showed partial responses to chemotherapy (response rate of $41.7 \%$ ). Grade 3-4 neutropenia developed in $10(83.3 \%)$ patients and grade 3 febrile neutropenia occurred in $5(41.7 \%)$ patients, but no grade 4 non-hematological toxicity was noted. Mortality associated with lung toxicity
\end{abstract}


(grade 5) due to treatment occurred in a 77-year-old-male patient with PS 3. No substantial difference in survival was observed between patients with PS 3 and 4, even when including those treated with supportive care alone. Treatment had a positive effect on survival: after chemotherapy, the 6 -month survival rate of PS 3 and 4 patients was $66.7 \%$. In contrast, all patients treated with supportive care alone died within 5 months. These findings suggest that chemotherapy is indicated in selected SCLC patients not only with PS 3 , but also with PS 4.

\section{Introduction}

Small-cell lung cancer (SCLC) accounts for about 13\% of all lung cancers in Japan, although its incidence has been gradually declining [1]. As it spreads rapidly to other body parts, more than $60 \%$ of newly diagnosed SCLC patients present extensive disease [2]. Moreover, the prognosis of these patients is very poor with a median survival of only 1-2 months on supportive care alone [3].

Ever since a significant survival benefit with cyclophosphamide monotherapy was shown in a randomized trial in 1960s [4], chemotherapy has been universally used to treat SCLC patients in a good general condition. Although SCLC is highly sensitive to platinum-based chemotherapy, its indication in patients with a poor performance status (PS, Eastern Cooperative Oncology Group [ECOG] PS 3 and 4) at initial diagnosis is still controversial, due to high treatment-related mortality risk of such patients $[5,6]$.

There has been only one randomized trial that evaluated platinum-based chemotherapy for poor-risk SCLC treatment in PS 3 patients $<70$ years of age) and PS 0-2 (ECOG, good PS) patients $\geq 70$ years of age. In this study, a subgroup analysis showed a median overall survival of 7.1 months in the patients with PS 3 treated with carboplatin and etoposide, and of 6.9 months in those treated with cisplatin and etoposide, without death occurrence due to toxicity of either treatment [7]. Regarding the results of a retrospective case series, the objective response rate to combined platinum and etoposide chemotherapy was $67 \%$ and the median overall survival was 2.1 months in patients with PS 3, which corresponded to $20 \%$ and 7 days, respectively, in those with PS 4 . Additionally, $20 \%$ of PS 3 patients and $60 \%$ of PS 4 patients died shortly after the first chemotherapy cycle [8]. Moreover, in another case series study of the effect of chemotherapy on poor-risk SCLC, the objective response rate and median overall survival were $75 \%$ and 8.4 months, respectively, in patients with PS 3, in contrast to $40 \%$ and 4.8 months, respectively, in those with PS 4 [9]. In addition, one patient with each PS died due to chemotherapy-related toxicity [9]. All these studies show discrepant results, which may be attributable to differences in patients' characteristics between clinical trials and clinical practice. Nonetheless, this issue should be addressed carefully to achieve a standard clinical treatment for such debilitated patients. Furthermore, only patients who received chemotherapy were included in these studies, however, best supportive care in clinical settings is also an alternative treatment to consider.

Therefore, the main objective of our retrospective case series study was to evaluate the clinical courses of extensive SCLC patients with poor PS at initial diagnosis in order to identify optimal treatment options. 


\section{Case Reports in Oncology}

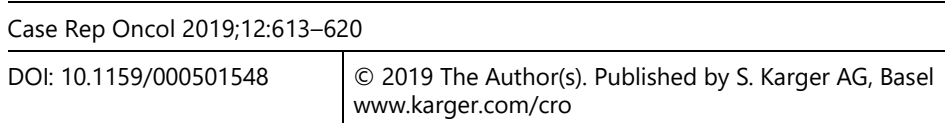

Aida et al.: Small-Cell Lung Cancer Treatment of Newly Diagnosed Patients with Poor Performance Status

\section{Patients and Methods}

We conducted a retrospective review of the clinical data of 18 pathologically diagnosed SCLC patients with poor PS, ECOG PS 3 and 4, treated at University of Tsukuba Hospital between April 2000 and May 2017.

The data collected from medical records of our hospital were as follows: medical history, physical examination on admission, pathological diagnosis, laboratorial and radiological findings, treatment regimen and respective adverse events/toxicity, ECOG PS grade variation (before and during chemotherapy), and patient's clinical course.

Furthermore, tumor responses to treatment were evaluated according to World Health Organization response criteria [10]. Treatment toxicities were evaluated according to Common Terminology Criteria for Adverse Events version 4.0.

The overall survival was determined from the pathological diagnosis to patient's death or last follow-up.

Statistical analyses were performed with Statistical Package for Social Sciences Soft Ware version 25.0. Survival curves were estimated by the Kaplan-Meier method and compared by the log rank test.

\section{Results}

\section{Patient Characteristics}

Among 142 patients diagnosed with SCLC by cytological or histological examination at out hospital, 12 had a PS 3 and 6 had a PS 4 at initial diagnosis. Table 1 summarizes the characteristics of the patients considered for study. Overall, 14 males and 4 females were included, with a median age of 73 years. There were no large differences in age, gender, or tumor-related factors between patients with PS 3 and 4.

\section{Treatment}

Twelve patients, with both PS3 and 4, were treated with chemotherapy and 6 patients with best supportive care alone (Table 1). Chemotherapy was not administered in 2 patients with PS3 due to severe comorbidities and mental disease. The chemotherapeutic regimen was a combination of carboplatin and etoposide in 11 out of those 12 patients. Of these, 7 patients received the standard dose, carboplatin area under the curve (AUC) of 5 intravenously on day 1 and etoposide $80-100 \mathrm{mg} / \mathrm{m}^{2}$ intravenously on days $1-3$. In the remaining 4 patients, the doses of carboplatin and etoposide were reduced to AUC of 4 and $50-80 \mathrm{mg} / \mathrm{m}^{2}$, respectively. One patient was only treated with carboplatin AUC of 5 because the serum total bilirubin level was high $(>5 \mathrm{mg} / \mathrm{dL})$ and he presented hepatic metastases. The number of chemotherapy cycles in the first-line chemotherapy ranged from 1 to 4 (50\% of patients underwent 4 cycles; $8.3 \%, 3 ; 8.3 \%, 2 ; 33.3 \%, 1$ ). Second-line chemotherapy was administered to 5 patients.

Whole brain radiation therapy was administered to 4 patients before chemotherapy and stereotactic radiotherapy for brain metastasis was administered to 1 patient. Additionally, 1 patient underwent palliative thoracic radiotherapy at a dose of 30.6 Gy in 17 fractions. 


\section{Improvement of PS}

During chemotherapy, PS improved in 7 (58.3\%) patients. This was observed in the 2 patients with PS 4 (Table 1): 1 patient shifted to PS 2, and the other shifted to PS 1 . In addition, PS remained unchanged in $2(16.7 \%)$ patients, while it deteriorated in $3(25 \%)$ patients (Fig. 1).

\section{Toxicity}

Grade 3-4 neutropenia developed in 10 (83\%), and grade 3 febrile neutropenia in 5 (42\%) patients (Table 2). Treatment-related mortality associated with lung toxicity occurred in a 77-year-old male patient with PS 3 . There were 3 early deaths within 4 weeks of treatment; these patients were all treated with supportive care alone and died of lung cancer progression.

\section{Objective Response and Overall Survival}

Considering all 18 patients studied, the median overall survival and 6-month survival rate were 4.9 months and $47.9 \%$, respectively. The overall survival of the patients with PS 4 was comparable to that of the patients with PS 3; the 6-month survival rate was $50 \%$ in both groups (log rank $p=0.31$, Fig. 2 ).

During chemotherapy, there were 5 partial responses (objective response rate of $41.7 \%$ ), 3 stable disease (25\%), and 4 progressive disease (33.3\%). Treatment significantly affected survival: the 6-month survival rate was $66.7 \%$ in those patients treated with chemotherapy, while all patients treated with supportive care alone died within the first 5 months (logrank $p=0.037$, Fig. 3).

\section{Discussion}

It is difficult to select the best treatment option in SCLC patients with a poor PS because occasionally chemotherapy is too toxic to be administered to patients in such a poor general condition. In this study, one patient (aged 77 years) with PS 3 died of chemotherapy, which might be explained by the patient's advanced age. Although toxicity was tolerable in the other patients studied (Table 2), it was reported that both poor PS and advanced age are significant factors associated with toxic death in patients with SCLC [5], as well as in those with breast and non-small-cell lung cancers [6].

On the contrary, patient's general condition may actually take a turn for the better when active systemic chemotherapy is administered. Indeed, in this study, a considerable improvement in PS after chemotherapy was observed in 7 of 12 (58\%) patients. This is comparable to the response rate to carboplatin and etoposide in elderly patients with extensive SCLC reported in a phase III trial [7]. Thus, it is reasonable to expect a PS improvement with systemic chemotherapy in SCLC having a poor PS.

The current Japanese lung cancer guidelines provide the treatment options for SCLC patients with PS 3 and 4 separately [11]. According to those, platinum doublets are recommended for patients with PS 3. However, this is based on only one phase III trial, which included 18 patients with PS 3 . In addition, this study excluded elderly patients $>70$ years of age, but currently this group corresponds to about half of the patients with a poor PS. Regarding 
patients with PS 4, the guidelines do not recommend chemotherapy because there are no available supportive data. However, we report on two patients with PS 4 in whom carboplatin and etoposide combined chemotherapy was so effective that their PS improved significantly. Accordingly, they were able to receive a total of four cycles of chemotherapy without lifethreatening toxicity. Although our number of patients was limited, overall survival did not differ between patients with PS 3 and 4, even when the patients treated with supportive care alone were included in the analysis. Importantly, the American guidelines for the treatment of SCLC do not consider patients with PS 3 and 4 separately, and recommend chemotherapy for all if the poor PS is a result of the SCLC itself [12-14]. Therefore, our findings strongly suggest that chemotherapy is indicated in selected SCLC patients with both PS 3 and 4 if the poor PS is mostly due SCLC rather than by comorbidities, as an improvement of PS due to treatment is expected.

In conclusion, the poor PS was improved significantly in about $58 \%$ of patients with aggressive systemic chemotherapy, strongly suggesting that chemotherapy is indicated in selected SCLC patients with both PS 3 and 4.

\section{Statement of Ethics}

This study was approved by the Ethical Committee of University of Tsukuba Hospital.

\section{Disclosure Statement}

The authors have no conflicts of interest to declare.

\section{References}

1 Toyoda Y, Nakayama T, Ioka A, Tsukuma H. Trends in lung cancer incidence by histological type in Osaka, Japan. Jpn J Clin Oncol. 2008 Aug;38(8):534-9.

2 Shepherd FA, Crowley J, Van Houtte P, Postmus PE, Carney D, Chansky K, et al. The International Association for the Study of Lung Cancer lung cancer staging project: proposals regarding the clinical staging of small cell lung cancer in the forthcoming (seventh) edition of the tumor, node, metastasis classification for lung cancer. J Thorac Oncol. 2007 Dec;2(12):1067-77.

3 Zelen M. Keynote address on biostatistics and data retrieval. Cancer Chemother Rep 3. 1973 Mar;4(2):31-42.

4 Green RA, Humphrey E, Close H, Patno ME. Alkylating agents in bronchogenic carcinoma. Am J Med. 1969 Apr;46(4):516-25.

5 Lassen UN, Osterlind K, Hirsch FR, Bergman B, Dombernowsky P, Hansen HH. Early death during chemotherapy in patients with small-cell lung cancer: derivation of a prognostic index for toxic death and progression. Br J Cancer. 1999 Feb;79(3-4):515-9.

6 Wallington M, Saxon EB, Bomb M, Smittenaar R, Wickenden M, McPhail S, et al. 30-day mortality after systemic anticancer treatment for breast and lung cancer in England: a population-based, observational study. Lancet Oncol. 2016 Sep;17(9):1203-16.

7 Okamoto H, Watanabe K, Kunikane H, Yokoyama A, Kudoh S, Asakawa T, et al. Randomised phase III trial of carboplatin plus etoposide vs split doses of cisplatin plus etoposide in elderly or poor-risk patients with extensive disease small-cell lung cancer: JCOG 9702. Br J Cancer. 2007 Jul;97(2):162-9. 
8 Baldotto CS, Cronemberger EH, de Biasi P, Zamboni M, Sousa A, et al. Palliative care in poor-performance status small cell lung cancer patients: is there a mandatory role for chemotherapy? Support Care Cancer. 2012 Nov;20(11):2721-7.

9 Sakuragi T, Oshita F, Nagashima S, Kasai T, Kurata T, Fukuda M, et al. Retrospective analysis of the treatment of patients with small cell lung cancer showing poor performance status. Jpn J Clin Oncol. 1996 Jun;26(3):128-33.

10 World Health Organization. WHO Handbook for Reporting Results of Cancer Treatment. WHO Publication No. 48 ed. Geneva; 1979.

11 The Japan Lung Cancer Society. Guidelines for Diagnosis and Treatment of the Lung Cancer 2018. ed. Tokyo: KANEHARA \& CO., LTD.; 2018 (in Japanese).

12 Jett JR, Schild SE, Kesler KA, Kalemkerian GP. Treatment of small cell lung cancer: Diagnosis and management of lung cancer, 3rd ed: American College of Chest Physicians evidence-based clinical practice guidelines. Chest. 2013 May;143(5 Suppl):e400S-19S.

13 Rudin CM, Ismaila N, Hann CL, Malhotra N, Movsas B, Norris K, et al. Treatment of Small-Cell Lung Cancer: American Society of Clinical Oncology Endorsement of the American College of Chest Physicians Guideline. J Clin Oncol. 2015 Dec;33(34):4106-11.

14 NCCN Clinical Practice Guidelines in Oncology. Small Cell Lung Cancer. Available at: https://www.nccn.org/professionals/physician_gls/. 2018.

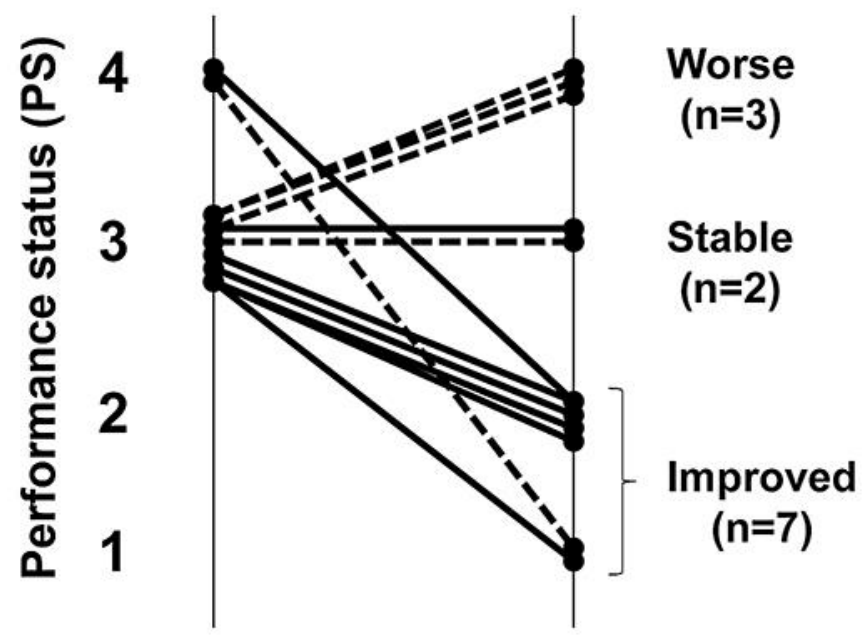

\section{PS before chemotherapy}

Fig. 1. Performance status (PS) variation during chemotherapy courses. As a result of treatment, PS improved in 7 (58.3\%), remained stable in 2 (16.7\%), and deteriorated in 3 (25\%) patients. Solid lines: patients who received standard chemotherapy; Dashed lines: patients who received chemotherapy at low doses; PS is categorized as 1-4. 


\section{Case Reports in Oncology}

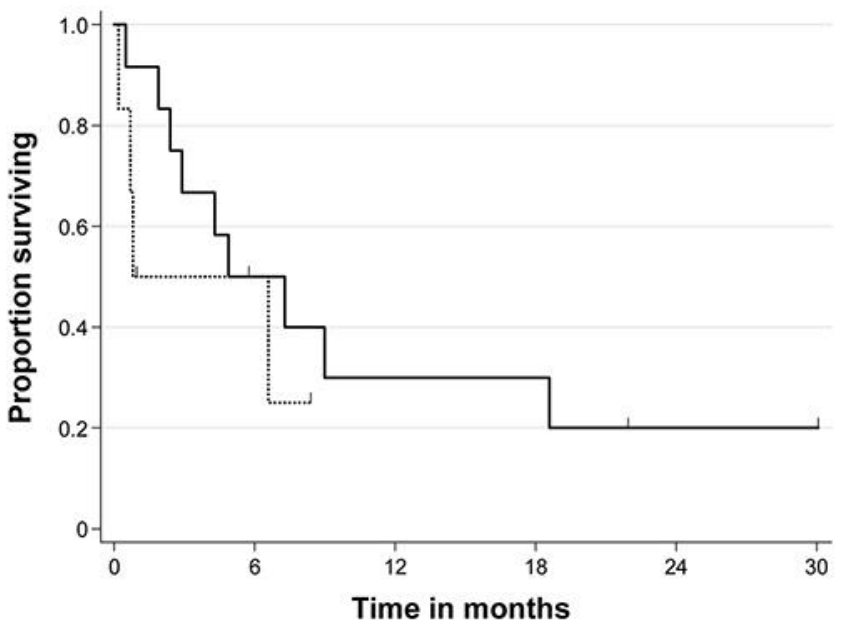

Fig. 2. Patients' overall survival according to performance status (PS). Solid line: patients with PS 3 ( $n=$ 12); Dotted line: patients with PS $4(n=6)$.

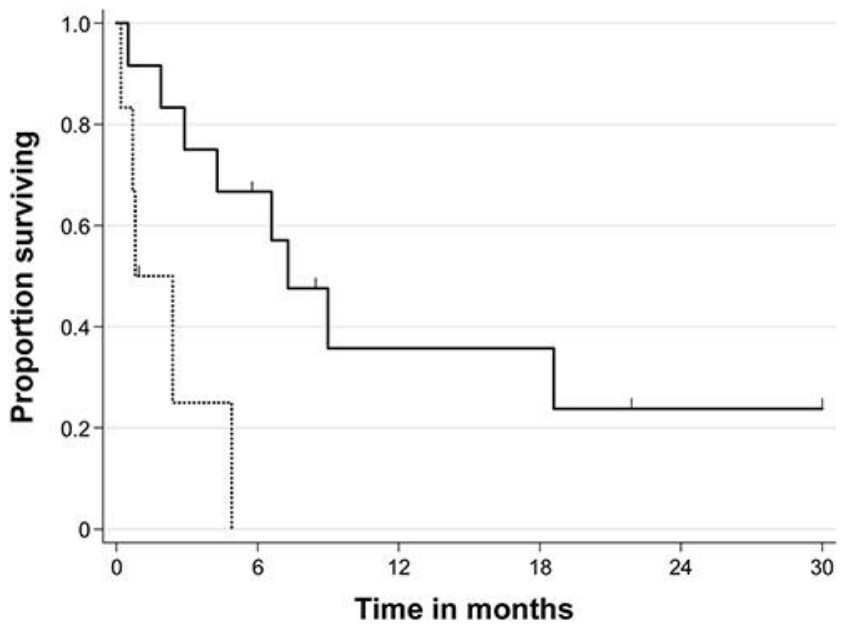

Fig. 3. Patients' overall survival according to treatment type. Solid line: patients treated with chemotherapy $(n=12)$; Dotted line: patients treated with supportive care alone $(n=6)$. 
Table 1. Patients' characteristics

\begin{tabular}{|c|c|c|c|}
\hline & PS $3(n=12)$ & PS $4(n=6)$ & Total $(n=18)$ \\
\hline \multicolumn{4}{|l|}{ Age, years } \\
\hline Median (range) & $74.5(51-85)$ & $70.5(64-77)$ & $73(51-85)$ \\
\hline \multicolumn{4}{|l|}{ Gender, $n(\%)$} \\
\hline Male & $8(66.7)$ & $6(100)$ & $14(77.8)$ \\
\hline Female & $4(33.3)$ & 0 & $4(22.2)$ \\
\hline \multicolumn{4}{|c|}{ Superior vena cava syndrome, $n(\%)$} \\
\hline Yes & 2 (16.7) & $1(16.7)$ & $3(16.7)$ \\
\hline No & $10(83.3)$ & $5(83.3)$ & $15(83.3)$ \\
\hline \multicolumn{4}{|l|}{ Brain metastases, $n(\%)$} \\
\hline Yes & $6(50)$ & $3(50)$ & $9(50)$ \\
\hline No & $6(50)$ & $2(33.3)$ & $8(44.4)$ \\
\hline Unknown & 0 & $1(16.7)$ & $1(5.6)$ \\
\hline \multicolumn{4}{|l|}{ Serum LDH, IU/L } \\
\hline Median (range) & $263(170-877)$ & $597.5(205-5,739)$ & $352(170-5,739)$ \\
\hline \multicolumn{4}{|l|}{ Treatment, $n(\%)$} \\
\hline Chemotherapy & $10(83.3)$ & $2(33.3)$ & 12 (66.7) \\
\hline Supportive care alone & 2 (16.7) & $4(66.7)$ & $6(33.3)$ \\
\hline
\end{tabular}

Table 2. Toxicity of first-line chemotherapy (Grade 3-5)

\begin{tabular}{|c|c|c|c|c|}
\hline & \multicolumn{4}{|c|}{ Toxicity, $n(\%)$} \\
\hline & grade 3 & grade 4 & grade 5 & grade $3-5$ (total) \\
\hline Neutropenia & $4(33.3)$ & $6(50)$ & 0 & $10(83.3)$ \\
\hline Anemia & $4(33.3)$ & $1(8.3)$ & 0 & $5(41.7)$ \\
\hline Thrombocytopenia & $5(41.7)$ & $1(8.3)$ & 0 & $6(50)$ \\
\hline Febrile neutropenia & $4(33.3)$ & 0 & 0 & $4(33.3)$ \\
\hline Dyspnea & 0 & 0 & $1(8.3)$ & $1(8.3)$ \\
\hline
\end{tabular}

$n$, number of patients. 